\title{
Experimental study of electromagnetic Bessel-Gaussian Schell Model beams propagating in a turbulent channel
}

\author{
S. Avramov-Zamurovic ${ }^{1 *}$, C. Nelson ${ }^{2}$, S. Guth ${ }^{3}$, O. Korotkova ${ }^{4}$ and R. Malek-Madani ${ }^{3}$ \\ ${ }^{I}$ Weapons and Systems Engineering, US Naval Academy, 105 Maryland Avenue, Annapolis, MD 21402, USA \\ ${ }^{2}$ Electrical and Computer Engineering Department, US Naval Academy, 105 Maryland Avenue, Annapolis, MD 21402, USA \\ ${ }^{3}$ Department of Mathematics, US Naval Academy, 589 McNair Rd, Stop 10M, Annapolis MD 21402, USA \\ ${ }^{4}$ Department of Physics, University of Miami, 1320 Campo Sano Dr., Coral Gables FL 33146, USA \\ *avramov@usna.edu
}

\begin{abstract}
We report on experimental generation of Electromagnetic Bessel-Gaussian Schellmodel [EBGSM] beams via incoherent superposition of two mutually orthogonal electric field components, both originated from a laser source, whose phases are spatially modified by two nematic liquid crystal Spatial Light Modulators. The EBGSM beam is then passed through a weakly fluctuating turbulent channel and examined for contrast in its fluctuating intensity. It is demonstrated that after passing through turbulence the electromagnetic beam exhibits reduction in the scintillation index on the order of $50 \%$, as compared with that for an equivalent scalar beam, in strong agreement with recent theoretical predictions.
\end{abstract}

Key words: spatially partially coherent laser beams; scintillation index; propagation of electromagnetic laser beams in turbulence

\section{Introduction}

The damaging effects of atmospheric turbulence on optical beam propagation can be mitigated through employment of various techniques: source partial coherence [1-5], aperture averaging [6], sparse aperture detectors [7, 8], wavelength diversity [9] and source temporal variations [10]. Additionally, it was recently proposed through analytical calculations [11] and simulations $[12,13]$ that polarization diversity can be effective in atmospheric mitigation as well. In particular, in [12] a beam generated by a deterministic electromagnetic source as a combination of two Laguerre modes showed considerable scintillation reduction with certain choices of mode parameters. Further, it was established in $[11,13]$ that a combination of partial coherence and partial polarization leads to even greater reduction in scintillation than these means of diversity applied separately. In the limiting case of a completely unpolarized beam the reduction in scintillation has been proven to be $1 / 2$, regardless of the coherence properties of the source and atmospheric link conditions.

So far, the only experimental verification of this result was obtained in the laboratory with the help of a temperature-controllable hot-air turbulence emulator [14]. The beams used for this purpose were the Multi-Gaussian Schell Model (MGSM) beams that form flat top beam intensity profiles in the far field of the source, being convenient for the detection procedure. The scalar [15-16] and electromagnetic [17] MGSM beams can be generated with the help of an SLM [14], [18] or with a hologram and a diffuser [19].

The aim of this paper is to extend the experimental results of Ref. [14] from turbulence emulator to actual atmospheric channel and to employ another class of electromagnetic partially 
coherent beams for confirming that the scintillation reduction outcome is source-independent. For this we generate the Bessel-Gaussian SchellModel (BGSM) beam that forms a ring-like intensity cross-section in the far field of the source [20] - [22].

Figure 1 shows a typical transverse cross-section of a well-formed scalar BGSM beam. In order to experimentally produce a scalar BGSM beam a sequence of statistically independent phase screens are cycled on a Spatial Light Modulator (SLM). By doing so, we separately generate horizontally and vertically polarized scalar beams from a single laser source and combine them incoherently into an electromagnetic beam. The spatial partial coherence for horizontal and vertical beams is obtained by two reflective SLMs. Their phase screen realizations belong to the same class, but are cycled independently. Hence, even though each SLM cycles the same set of phase screens, the combination of the beams leads to unpolarized beam, provided the power levels in both polarizations are the same.

In order to prove the $50 \%$ reduction in the scintillation index we compare a) horizontally $(\mathrm{H})$; b) vertically (V) polarized BGSM scalar beams, and c) a BGSM beam obtained as an incoherent superposition of $(\mathrm{H})$ and $(\mathrm{V})$ beams, referred here as $(\mathrm{HV})$ beam. While $(\mathrm{H})$ and $(\mathrm{V})$ beams can be viewed as scalar, (HV) beam is fundamentally electromagnetic, provided that the intensities in $(\mathrm{H})$ and $(\mathrm{V})$ are equal (HV) beam is ideally unpolarized.

This paper is organized as follows. First, the theoretical background for generating spatially partially coherent scalar beams using the SLM phase screens is presented. Second, the experimental set-up for the realization of various beams is described and a detailed description of the instrumentation and data processing methods are given. Third, details of the procedure to remove background noise from images of fluctuating light intensities and the calculations of the scintillation index are provided. Fourth, an estimation of the refractive index structure parameter of atmospheric fluctuations $C_{n}{ }^{2}$ is given to provide information on the turbulent regime. Finally, the results section summarizes the performance of various beams with regards to their scintillation index. For quantitative analysis of observed BGSM beams, the spatial distributions of scintillations and their dependence on time and space averaging are presented.

\section{Theoretical background}

Since this paper emphasizes experimental study, we will only review the theory that motivated our research. We first present the source coherence properties of the BGSM beams. Next, we discuss the simulation of these beams in the far field by using a) theoretical models and b) phase modulation limitation of SLM used to produce them. Finally, we provide the definition of the scintillation index for the beams with uncorrelated electric field components.

\section{BGSM beams source spectral degree of coherence}

A newly developed model for the BGSM (ring) beams, gives the following spectral (scalar) degree of coherence [20] at the source: 


$$
\eta^{(0)}\left(\rho_{1}-\rho_{2}\right)=J_{0}\left(\beta \frac{\left|\rho_{1}-\rho_{2}\right|}{\alpha}\right) e^{\left(\frac{\left|\rho_{1}-\rho_{2}\right|^{2}}{2 \alpha^{2}}\right)},
$$

Where $J_{0}(x)$ is the Bessel function of the first kind and zero order, and $\rho_{1}=\left|\boldsymbol{\rho}_{1}\right|, \rho_{2}=\left|\boldsymbol{\rho}_{2}\right| \rho=$ $\left|\rho_{1}-\rho_{2}\right|$ are position distances and superscript (0) refers to the source plane. $\beta$ and $\alpha$ are real numbers that control the radius and the thickness of the ring. To generate the BGSM beams, we selected parameters $\beta=4$ and $\alpha=4$. These parameters were chosen to best represent balanced ring size and thickness. Other parameter combinations were tested and this set proved to best serve the purpose of our experiment where the scintillation index was measured for $\mathrm{H}, \mathrm{V}$, and $\mathrm{HV}$ beams.

\section{BGSM beams simulation}

Simulation of our projected experimental beams shows the BGSM beam cross section in the far field (Fig. 1). It was created using 100 phase-screen realizations prescribed by equations 2.1.

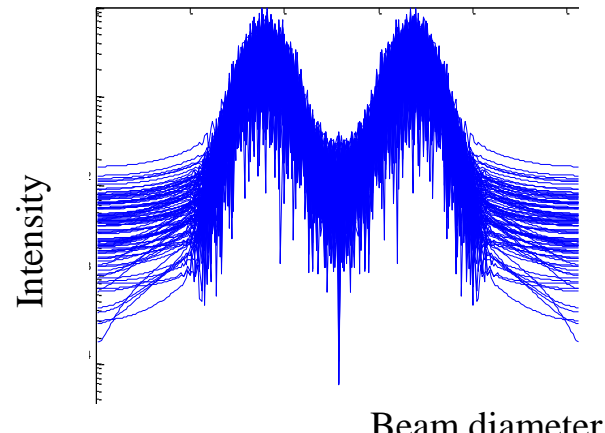

a)

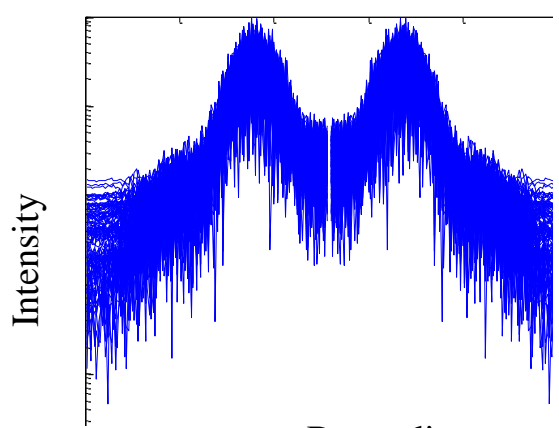

Beam diameter

b)

Fig.1. Computer simulated BGSM beam cross-section in the far field. BGSM beams a) theoretical predictions and b) predictions using the SLM model. Intensity is given in logarithmic scale. The center pixel was removed in b) and the values were normalized. This is a qualitative representation of the beams used in our experimentation.

By setting up a suitable screen on an SLM, one can generate an arbitrary beam shape [18]. Our investigation focuses on beams with a ring shape that have sloping edges [20]. Theoretically, a far field intensity pattern is obtained by modulating the amplitude and phase of the incoming laser beam according to the prescribed screen values. Practically, the SLM can only modulate the phase of the incoming light, and preserves the amplitude of the incoming (Gaussian) beam. However, as was shown in [23] (see also [18]) the programming of the SLM can be slightly adjusted in order for phase-only spatial distribution to approximate both amplitude and phase distributions. Slight differences between the theoretical and practical realizations are visible on Fig. 1. Images of the beams experimentally were accomplished using the SLMs corresponding with the patterns given in Figs. 1(b). 
The following explains the differences between the theoretical realizations and practical estimates of the partially coherent beams in the far field. Let $\mathrm{N}(\mathrm{x}, \mathrm{y} ; 0,1)$ be the Normal Gaussian distribution matrix representing spatially distributed (in $\mathrm{x}$ and $\mathrm{y}$ ) uncorrelated random noise. The partially spatially coherent beam screen, $S_{t}(x, y)$ is equal to the convolution of the spectral degree of coherence matrix, $\mu^{(0)}(\mathrm{s})$, with the noise array $\mathrm{N}(\mathrm{x}, \mathrm{y} ; 0,1)$. The theoretical representation of the far field beam profile is given by the Fourier Transform of the screen $S_{t}(x, y)$, as given in Fig. 1(a). This plot is acquired using both phase and amplitude modulation of the beam. In the case of the SLM, which is capable of only modulating the phase of the incoming beam, the plot in Fig. $1(\mathrm{~b})$ is obtained by finding the Fourier transform of $e^{\mathrm{St}(\mathrm{x}, \mathrm{y})}[23]$. The Fourier transform of $e^{\mathrm{St}(\mathrm{x}, \mathrm{y})}$ has a significant $\mathrm{DC}$ component that has been removed from the simulation plots, as it did not influence the data collection experimentally. This representation matches well with the actual experiment. Note that the intensities of the SLM estimates have a higher level of noise. This feature is faint, but observable at short propagation distances and it vanishes in the far field.

\section{Scintillation index of the electromagnetic beams with uncorrelated orthogonal field components}

The second-order correlation properties of a wide-sense statistically stationary electromagnetic beam can be described by means of the beam coherence-polarization matrix [24] or cross-spectral density matrix [25] whose spatial counterparts have the same form.

The conventional measure of the intensity fluctuations at a single position in an optical wave is its normalized variance or the scintillation index, defined as

$$
c(\mathbf{r})=\frac{i^{(I I)}(\mathbf{r})-\left[i^{(I)}(\mathbf{r})\right]^{2}}{\left[i^{(I)}(\mathbf{r})\right]^{2}}
$$

Where $i^{(I I)}(\boldsymbol{r})=\left\langle i(\boldsymbol{r})^{2}\right\rangle$ and $i^{(I)}(\boldsymbol{r})=\langle i(\boldsymbol{r})\rangle$ are the second and the first moment of the instantaneous intensity $i(\mathbf{r})$ and $\mathbf{r}$ is the position vector. As was shown in [11], the scintillation index of an electromagnetic beam may be expressed in the more general form:

$$
c(\mathbf{r})=\frac{c_{x x}(\mathbf{r})\left[i_{x}^{(I)}(\mathbf{r})\right]^{2}+2 c_{x y}(\mathbf{r}) i_{x}^{(I)}(\mathbf{r}) i_{y}^{(I)}(\mathbf{r})+c_{y y}(\mathbf{r})\left[i_{y}^{(I)}(\mathbf{r})\right]^{2}}{\left[i_{x}^{(I)}(\mathbf{r})+i_{y}^{(I)}(\mathbf{r})\right]^{2}}
$$

In this representation $i_{x}^{(I)}$ and $i_{y}^{(I)}$ are the intensities of $\mathrm{x}$ and $\mathrm{y}$ components of the electric field

while $c_{x x}(\mathbf{r}), c_{y y}(\mathbf{r})$ are the scintillation indexes of the field components fluctuating in two orthogonal directions and $c_{x y}(\mathbf{r})$ is that for their mutual intensity: 


$$
c_{x y}(\mathbf{r})=\frac{\left\langle i_{x}(r) i_{y}(r)\right\rangle-i_{x}^{(I)}(r) i_{y}^{(I)}(r)}{i_{x}^{(I)}(r) i_{y}^{(I)}(r)}
$$

An important result relating to fields with uncorrelated field components immediately follows from formula (2.3): $c_{x y}(\mathbf{r})$ vanishing leads to a reduction in the scintillation index compared to that for fully or partially correlated field components. In the limiting case of an unpolarized light beam, i.e., that with uncorrelated electric field components with equal intensities $i_{x}=i_{y}$ the scintillation index can be readily shown to be reduced by a factor of two, compared to an equivalent polarized beam [11].

The reduction of the scintillation index was found using the following formula

$$
R=\frac{\frac{c_{x x}(\boldsymbol{r})+c_{y y}(\boldsymbol{r})}{2}-c(\boldsymbol{r})}{\frac{c_{x x}(\boldsymbol{r})+c_{y y}(\boldsymbol{r})}{2}}
$$

In our analysis, we utilized equation 2.3 to find the scintillation index of each scalar beam we propagated; namely horizontally or vertically polarized partially coherent beams. Equation 2.4 was used to calculate the scintillation index of the electromagnetic beams that we constructed by combining horizontally and vertically polarized beams, representing randomly polarized beam with uncorrelated beam components.

\section{Experiemental set up}

Figure 2 shows the experimental set-up used to generate and propagate spatially partially coherent unpolarized beams (compare with suggested setups in [26]- [29] and the previous setup used by the authors [14]). A $2 \mathrm{~mW}$ HeNe linearly polarized laser source generates a Gaussian beam with a diameter of $0.5 \mathrm{~mm}$. A beam expander was utilized to expand the beam to fill the SLM window. The expanded beam was then split through use of a 50/50 beam splitter in order to create vertically and horizontally polarized branches. In the horizontal branch a mirror and quarter-wave plate were inserted to rotate the vertically polarized beam from the laser source. The beam was then reflected from the SLM and sent through a linear polarizer. The horizontally polarized beam was then reflected from a mirror and passed through a 50/50 Pellicle beam splitter before entering the propagation channel. The vertical branch was similarly structured but physically oriented vertically; it additionally had a neutral density filter to adjust the power levels.

When the beams in the two branches are incoherently superimposed, a single unpolarized beam is formed. Two computers are used to control the SLMs. The beam propagates through a corridor and at the distance of $74 \mathrm{~m}$ the light field is captured using a high speed camera. The beam illuminated the light sensor directly as shown in Fig. 4. 
The measurement procedure was as follows. First, the system alignment was performed using a polarimeter to adjust the orthogonality of two field components to within 89 degrees and beam overlap was carefully adjusted in the near and far fields to ensure co-propagation of the two beams. Next, the power levels in the horizontal and vertical branches were approximately matched to within a couple of $\mathrm{dB}$. Ring beam sizes were approximately matched as shown in Fig. 3. Note that the zeroth order mode visible on Fig. 3, which aided during the alignment procedure and did not influence the results.

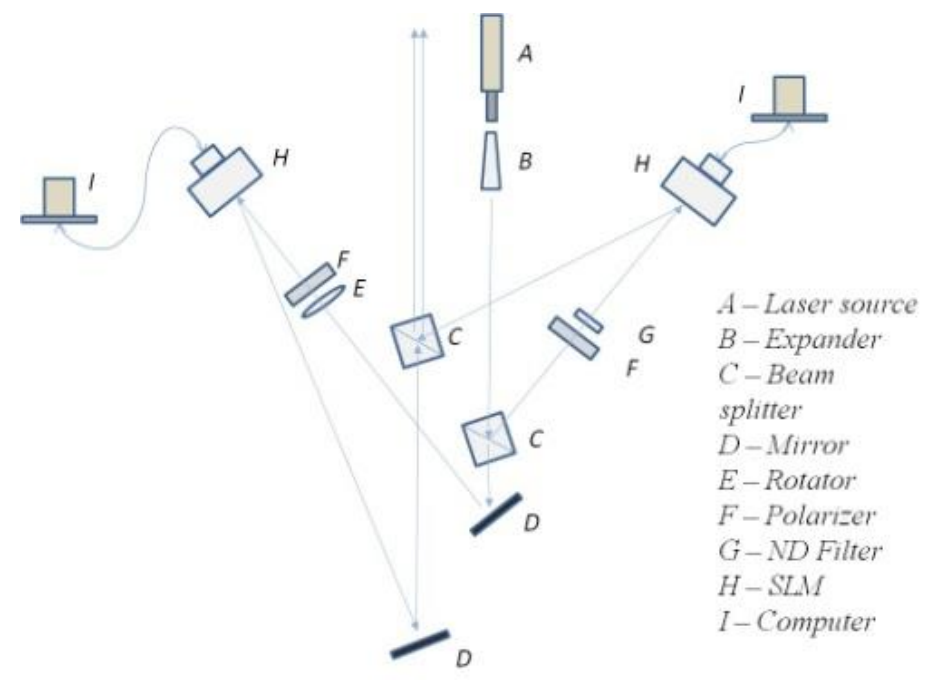

a)

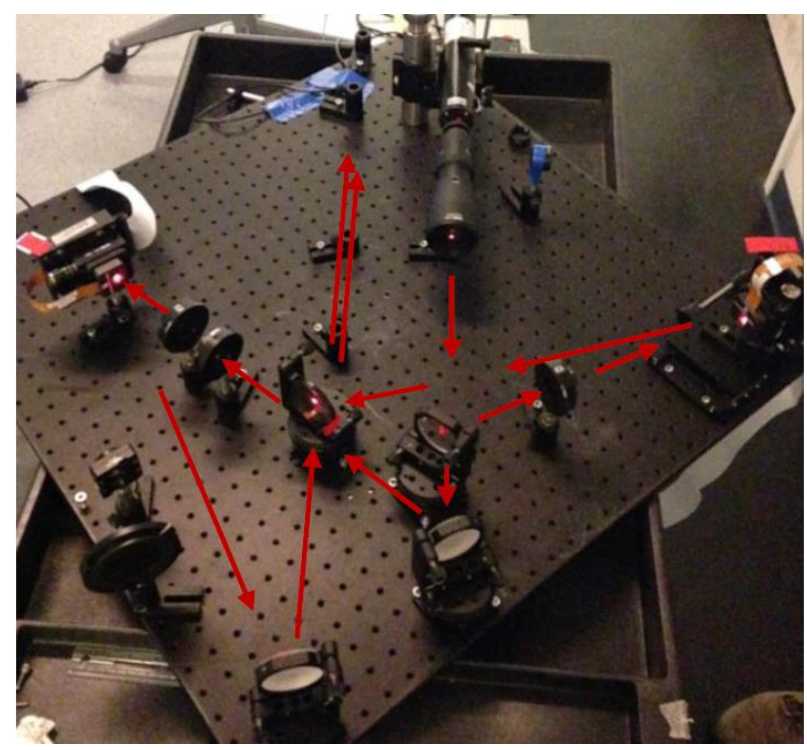

b) 
Fig. 2. General experimental set up for generating electromagnetic random beams. a) Schematic diagram and $b$ ) image of the experiment.

The camera sensor was placed directly in the path of the beam, and laser beam fluctuations were recorded through a red bandpass filter. Experimental data was collected for one minute per run, with runs cycling between vertical polarization only, horizontal polarization only, and combined beam. This set of three runs was repeated three or more times for each recorded gain setting on the camera. Following the data runs, the background level at each camera gain setting was measured by blocking the laser beam.

For each trial the same set of the SLM screens was used [see Eq. (2.1)] to create partially coherent beams. The phase screens for the combined beam were not synchronized in space or time, between vertical and horizontal branches. Camera gain was varied from run to run to establish the reliability of the process, and runs were repeated to add to fidelity of the analysis.

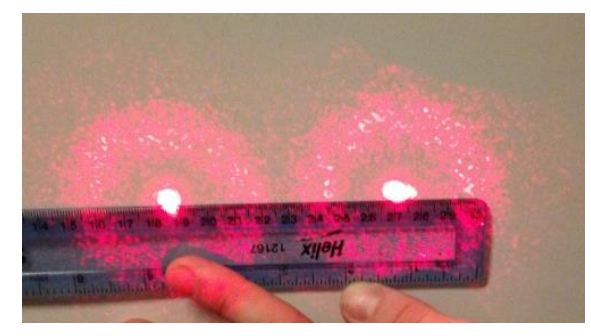

Fig. 3. BGSM beam rings size match at the distance of $\sim 3 \mathrm{~m}$.

The camera used in the experiments was configured to take 300 frames per second across the sensor area of $8.4667 \mathrm{~mm}$ x $8.4667 \mathrm{~mm}$. The camera sensor has $480 \times 640$ pixels, and in order to reach a high data throughput, camera hardware binning was utilized. This method averages the intensities of 24 pixels to produce a matrix of $20 \times 640$ values that cover the whole sensor area. This detail will show in the Results section of the paper in the form of long rectangular pattern in the figures that represent measured light intensities.

The two SLMs used in this experiment were identical devices with the same manufacturer specifications and the same resolution of spatial phase change; the SLM screen area is $6.14 \mathrm{~mm}$ x $6.14 \mathrm{~mm}$ with $256 \times 256$ pixels. The expansion of the beam due to the SLM pixelization is not significant for the analysis of laser beam across the light sensor presented in this paper. The SLMs were configured to change screen realizations at the rate of about $333 \mathrm{~Hz}$. Eight thousand SLM screen frames with the prescribed statistics [see Eq. 2.1] were cycled for each test run. 


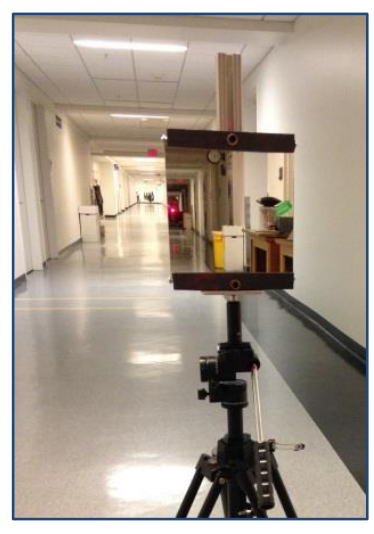

a)

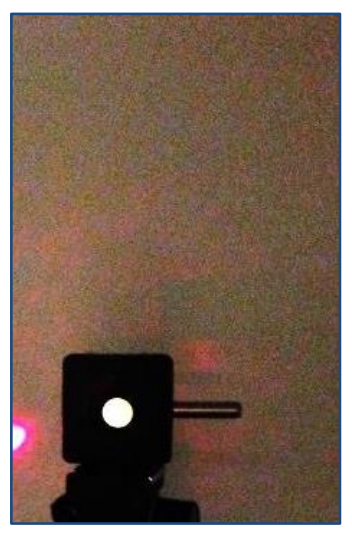

b)

Fig. 4. a) Mirror is located at half propagation distance, b) camera capture of beam after propagation and just to the side of the zeroth order mode. We utilized radial symmetry of the beams and as can be seen in Fig. $4 \mathrm{a}$, only one quadrant of the beam is reflected from the mirror to the detector.

\section{Data processing}

Our analysis goal is to calculate the scintillation index from the measured intensities of the laser beam as the ratio of the variance, $\sigma^{2}$, and the mean, $\mu$, squared. The first step is to express our intensity values in a three-dimensional array, $I_{\text {data }}(\mathbf{r}, t)$. We find the mean, $\mu_{t}(\mathbf{r})$, and the variance, $\sigma_{t}^{2}(r)$, of the set of the realizations, for each pixel to create the value $c(r)=\frac{\sigma_{t}^{2}(r)}{\mu_{t}^{2}(r)}$. Because the area of the camera sensor is small compared to the size scale of the evolved beam, we can compute a meaningful spatial average

$$
\langle c\rangle_{\mathbf{r}}=\left\langle\frac{\sigma_{\mathrm{t}}^{2}\left(\mathrm{I}_{\mathrm{data}}(\mathbf{r})\right)}{\mu_{\mathrm{t}}^{2}\left(\mathrm{I}_{\mathrm{data}}(\mathbf{r})\right)}\right\rangle_{\mathbf{r}}
$$

Notably, the $c(\mathbf{r})$ does display some spatial variation, due to the spatial inhomogeneity of the shaped beams, but we consider the spatial mean, $\langle c\rangle_{\mathbf{r}}$ a representation of the scintillation index across the sensor area. Equation 4.1 is a restatement of equation 2.2. in a form that is convenient for actual computations using experimental data.

\section{Dependence of scintillation index on background}

Due to the presence of the background noise we measured in addition to the laser signal, we introduce the following modification to Eq. 4.1 This approach is motivated by [30].

Suppose that

$$
I_{\text {data }}(\mathbf{r}, t)=\alpha(\mathbf{r}) I_{\text {desired }}(\mathbf{r}, t)+B(r, t)
$$

that is, our measured intensities, $I_{\text {data }}(r, t)$ are the sum of the beam, $\alpha(\mathbf{r}) I_{\text {desired }}(r, t)$ we intend to observe and some background, $\mathrm{B}(\mathbf{r}, \mathrm{t})$ noise [28]. This background may be caused by the camera's nonzero black level, camera readout noise, or reflections from the overhead lights in the 
corridor. The scale factor $\alpha(\mathbf{r})$ represents the conversion between incoming intensity (in physical units) and the camera output (a purely digital signal), which may vary across the camera's detector due to hardware. Then, assuming the independence between $\alpha(\mathbf{r}) \mathrm{I}_{\text {real }}(\mathbf{r}, \mathrm{t})$ and $B(\mathbf{r}, \mathrm{t})$,

$$
\left\langle c_{\text {data }}\right\rangle_{\mathbf{r}}=\left\langle\frac{\sigma_{\mathrm{t}}^{2}\left(\alpha(\mathbf{r}) \mathrm{I}_{\text {desired }}(\mathbf{r})\right)+\sigma_{\mathrm{t}}^{2}(\mathrm{~B}(\mathbf{r}))}{\mu_{\mathrm{t}}^{2}\left(\alpha(\mathbf{r}) \mathrm{I}_{\text {desired }}(\mathbf{r}, \mathrm{t})\right)+\mu_{\mathrm{t}}^{2}(\mathrm{~B}(\mathbf{r}))}\right\rangle_{\mathbf{r}}
$$

Where, the variance operator is linear in uncorrelated random variables, so we were able to separate out the numerator. By hypothesis, $\sigma_{t}^{2}\left(\alpha(\mathbf{r}) \mathrm{I}_{\text {desired }}(\mathbf{r})\right) \gg \sigma_{\mathrm{t}}^{2}(\mathrm{~B}(\mathbf{r}))$, so we ignore the second term in the numerator (the background variance). In our data, this inequality holds by many orders of magnitude. However, it is not clear that we can ignore the denominator (the background mean).

If we assume that $\mu_{\mathrm{t}}^{2}(\mathrm{~B}(\mathbf{r}))=0$, then $\left\langle\mathrm{c}_{\text {data }}\right\rangle_{\mathbf{r}}$ reduces to the scintillation index of the laser beam intensity we intended to measure $\left\langle\mathrm{c}_{\text {desired }}\right\rangle_{\mathbf{r}}$

$$
\left\langle c_{\text {data }}\right\rangle_{\mathbf{r}}=\left\langle\frac{\sigma_{\mathrm{t}}^{2}\left(\alpha(\mathbf{r}) \mathrm{I}_{\text {desired }}(\mathbf{r})\right)}{\mu_{\mathrm{t}}^{2}\left(\alpha(\mathbf{r}) \mathrm{I}(\mathbf{r})_{\text {desired }}(\mathbf{r})+0\right)}\right\rangle_{\mathbf{r}}=\left\langle c_{\text {desired }}\right\rangle_{\mathbf{r}}
$$

This derivation shows the insensitivity of the scintillation index to (even spatially dependent) scale factors. This is our justification for ignoring the absolute scale of the camera's intensity measurements.

However, suppose we increase $\mu_{t}(B(\mathbf{r}))$ until $\mu_{t}(B(r)) \sim \mu_{t}\left(\alpha(\mathbf{r}) I_{\text {desired }}(\mathbf{r})\right)$, that is, we increase the mean background until it has the same magnitude as the signal. We could do this by turning up the hallway lights, reducing the laser power, or tweaking the camera's black level. Crucially, for illustrative purposes we do this without significantly changing $\sigma_{\mathrm{t}}^{2}(\mathrm{~B}(\mathbf{r}))$. Then scintillation index takes the form

$$
\left\langle c_{\text {data }}\right\rangle_{\mathbf{r}}=\left\langle\frac{\sigma_{\mathrm{t}}^{2}\left(\alpha(\mathbf{r}) \mathrm{I}_{\text {desired }}(\mathbf{r})\right)}{\mu_{\mathrm{t}}^{2}\left(2 \alpha(\mathbf{r}) \mathrm{I}_{\text {desired }}(\mathbf{r})\right)}\right\rangle_{\mathbf{r}}=\frac{1}{4}\left\langle\mathrm{c}_{\text {desired }}\right\rangle_{\mathbf{r}}
$$

By this construction, we show the sensitivity of the scintillation index on the background mean. In summary the scintillation index is relatively insensitive to the absolute scale of the intensity measurements, but depends strongly on the background, $|B(r, t)|$, of the intensity measurements.

\section{Background removal}

This section describes our process for removing the background mean from the data. That is, we would like that $I_{\text {data }}=0$ implies that $I_{\text {desired }}=0$ and that $I_{\text {data }}\left(t_{1}\right)=\beta I_{\text {data }}\left(t_{2}\right)$ implies $I_{\text {real }}\left(t_{1}\right)=\beta I_{\text {desired }}\left(t_{2}\right)$, where $\beta$ is a scaling factor and $t_{1}$ and $t_{2}$ are two separate 
time instants in the same data run. Then we may be confident that $\left\langle\mathrm{c}_{\text {data }}\right\rangle_{\mathbf{r}}=\left\langle\mathrm{c}_{\text {desired }}\right\rangle_{\mathbf{r}}$. This is accomplished by measuring $\mathrm{I}_{\text {background }}$, the camera measurement of the background.

We measure $I_{\text {background }}$ with an otherwise identical procedure to our measurement of $I_{\text {data }}$ but with the laser completely blocked at the source. That is, we took a sequence of images of the corridor with no laser, and measured only camera readout noise, camera black value, and nonlaser light from the hallway. From these images, we calculated $\sigma_{\mathrm{t}}^{2}\left(\mathrm{I}_{\text {background }}(\mathbf{r})\right)$ and $\mu_{\mathrm{t}}\left(\mathrm{I}_{\text {background }}(\mathbf{r})\right)$. As expected, $\sigma_{\mathrm{t}}^{2}\left(\mathrm{I}_{\text {background }}(\mathbf{r})\right) \ll \sigma_{\mathrm{t}}^{2}\left(\mathrm{I}_{\text {data }}(\mathbf{r})\right)$, and subtracting the variance of the background from the beam variance made no difference to the results.

By linearity of $\mu_{\mathrm{t}}$, if we identify $\mu_{\mathrm{t}}\left(\mathrm{I}_{\text {background }}(\mathbf{r})\right)=\mu_{\mathrm{t}}(\mathrm{B}(\mathbf{r}))$ we expect that

$$
\mu_{\mathrm{t}}\left(\mathrm{I}_{\text {data }}(\mathbf{r})\right)-\mu_{\mathrm{t}}\left(\mathrm{I}_{\text {background }}(\mathbf{r})\right)=\alpha(\mathbf{r}) \mu_{\mathrm{t}}\left(\mathrm{I}_{\text {desired }}(\mathbf{r})\right)
$$

with some (spatially dependent) scale factor that does not influence the scintillation index calculations.

In summary, our data processing procedure is summarized as:

1) Measure $I_{\text {data }}(r, t)$ via camera images.

2) Measure $I_{\text {background }}(r, t)$ via camera images.

3) Compute $\mu_{t}\left(I_{\text {background }}(\mathbf{r})\right)$, the temporal mean background intensity

4) Compute $I_{\text {desired }}(\mathbf{r})=I_{\text {data }}(r, t)-\mu_{t}\left(I_{\text {background }}(\mathbf{r})\right)$, the background corrected data sequence, or the observed desired laser intensities fluctuations

5) Compute $c(\mathbf{r})=\frac{\sigma_{t}^{2}\left(I_{\text {desired }}(\mathbf{r})\right)}{\mu_{\mathrm{t}}^{2}\left(\mathrm{I}_{\text {rdesired }}(\mathbf{r})\right)}$, the spatially varying scintillation index

6) Compute $\langle c\rangle_{\mathrm{r}}$, the spatial average scintillation index across the sensor (camera) area due to the laser intensity fluctuations.

\section{Dependence of scintillation index on frame rate}

The calculated scintillation index has a strong dependence on the SLM cycling rate that can be explained in the following manner. Suppose that $R_{\text {cam }}=\frac{1}{k} R_{\text {SLM }}$, where $R_{\text {cam }}$ is the rate at which the camera takes images and $R_{\text {SLM }}$ is the rate at which SLM cycles the phase screens and $\mathrm{k}$ is a binning factor. If we let $\mathrm{k}=1$, then, each image records a distinct beam pattern directly related to the phase screen at the moment of recording. If $\mathrm{k}=2$, then the camera will average the light produced by two phase screens. Each camera pixel may record two dark spots, two light spots, or a light spot and a dark spot in succession.

If we assume that the dark and light spot distribution among the random phase screens follow a binomial distribution then as the binning factor, $\mathrm{k}$, grows, most pixels will see a mix of dark and light spots. We believe this is fair, because the sequential phase screens are uncorrelated. If 
we control for the effects of the increased camera exposure time on the recorded intensity, this growth in $\mathrm{k}$ will cause the temporal variance $\sigma_{\mathrm{t}}^{2}\left(\mathrm{I}_{\text {data }}(\mathbf{r})\right)$ to shrink.

In hardware, increasing the exposure time has the natural effect of temporal binning. However, we can emulate the effect of increased exposure time by binning in software. We can add k sequential frames together to create a new frame,

$$
I_{\text {bin-k }}(r, \hat{t})=\sum_{j=1, k} I_{d a t a}(r, t+j)
$$

and advance $\mathrm{t}$ by increments of $\mathrm{k}$.

When performing statistics on the temporally binned frames, we see a reduction in calculated scintillation, as expected. When we perform a similar binning procedure spatially (to emulate larger pixels), the observed change is small. (See Fig. 9).We interpret this to mean that the size of the far-field light and dark spots of the beam are large compared to the size of the camera pixels. Additionally, as spatial binning in software influences the calculated scintillation index slightly, we expect our spatial binning in hardware not to affect our results significantly. We find that the statistical noise averaging appears to compensate for the reduction in resolution [29].

\section{Characterization of the turbulence in the channel}

The experiments were conducted along an indoor corridor, approximately 7x5x180 meters in dimensions, while a commercial air conditioning system ran. To estimate the optical turbulence level, we assumed that the tests were conducted in the weak fluctuation regime and used the theoretical background given in [33].To obtain a data set necessary to estimate the refractive index structure parameter, we propagated a Gaussian laser beam reflected from a black (constant phase) phase screen and measured its intensity fluctuations. While we repeated this procedure with each possible polarization, there was little variation. The scintillation index was obtained from the measurements and used with other beam parameters to give an order of magnitude estimate of $C_{n}^{2}$.

The procedure utilized for $C_{n}^{2}$ estimation closely follows the derivation prescribed by [33] (pages 118,119, 140 and 351). First, the input and output plane parameters were calculated by using equations 5.1-5.3.

$$
\Lambda_{0}=\frac{2 \mathrm{z}}{k \mathrm{~W}_{0}^{2}}
$$

Where $\Lambda_{0}$ is input plane diffraction parameter, in our case the factors are distance $\mathrm{z}=74 \mathrm{~m}$, wave number $k=\frac{2 \pi}{\lambda}$ beam radius $\mathrm{W}_{0}=5 \mathrm{~mm} \lambda=633 \mathrm{~nm}$. 


$$
\Theta_{0}=1-\frac{\mathrm{z}}{F_{0}} \approx 1
$$

Where $\Theta_{0}$ is input plane refraction parameter, 1 for collimated beam and

$\Theta=\frac{\Theta_{0}}{\Theta_{0}^{2}+\Lambda_{0}^{2}}$ output plane refraction $\Lambda=\frac{\Lambda_{0}}{\Theta_{0}^{2}+\Lambda_{0}^{2}}$ output plane diffraction paramters

The scintillation index was calculated using the data processing method described in section 4 and labeled in this derivation as $\sigma_{B}^{2}$. The Rytov variance $\sigma_{R}^{2}$ was found from equation 5.3 by using an approximation of scintillation index $\sigma_{B}^{2}$.

$$
\sigma_{B}^{2} \cong \sigma_{R}^{2} 3.86\left\{0.40\left[(1+2 \Theta)^{2}+4 \Lambda^{2}\right]^{\frac{5}{12}} \cos \left[\frac{5}{6} \tan ^{-1}\left(\frac{1+2 \Theta}{2 \Lambda}\right)\right]-\frac{11}{16} \Lambda^{\frac{5}{6}}\right\}
$$

Note that the coefficient multiplying the $\sigma_{R}^{2}$ is 1.046 , justifying the plane wave approximation used in our estimation. Finally $C_{n}^{2}$ was estimated using equation 5.4 [33] to be at the order of $\sim 10^{-14}$.

$\sigma_{R}^{2}=1.23 C_{n}^{2} k^{\frac{7}{6}} L^{\frac{11}{6}}$

Spatial distribution of the estimated $C_{n}^{2}$ values across the sensor area is given in Fig. 5. The pattern does not show significant variation between the electromagnetic (combined) and horizontally polarized Gaussian beam. The vertically polarized beam has a slightly higher scintillation, possibly due to air flow from the air-conditioning system.

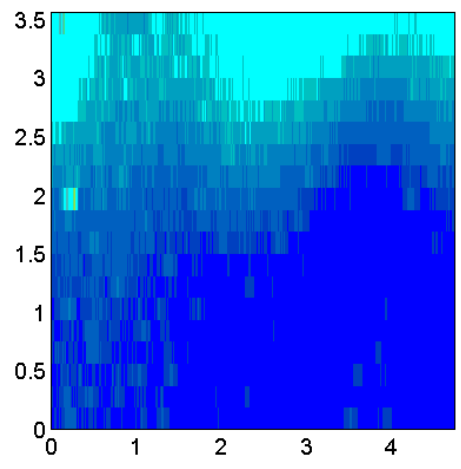

a)

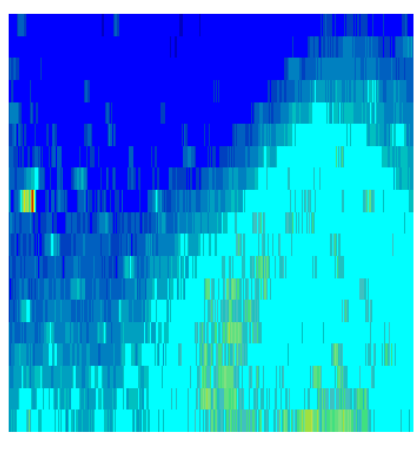

b)

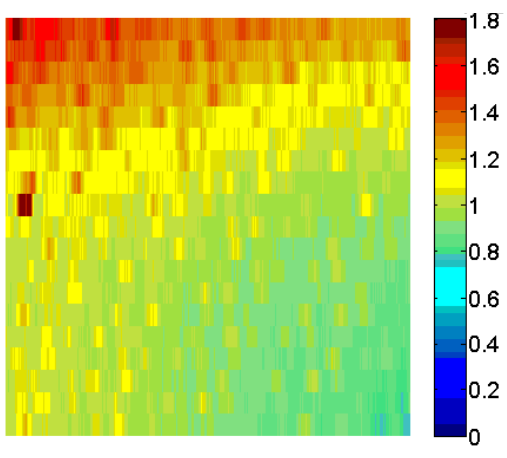

c)

Fig. 5. Estimated $C_{n}^{2}$ spatial distribution across the sensor's area (units are $10^{-13}$ ). a) Combined HV beam b) Horizontally polarized, c) Vertically polarized beam. Axes are spatial positions in $\mathrm{mm}$.

\section{Results}


The summary of the measurement data analysis for a comprehensive set of experiments is given in Table 1. The BGSM beams were propagated in similar environmental conditions and all tests were performed sequentially. To eliminate measurement bias, background light was recorded and used in the data processing procedures as given in section 4 . From previous experience, a wide range of intensities were expected for the different beam spatial patterns at the end of the $74 \mathrm{~m}$ link. In order to eliminate the camera recording gain bias, several gain values were tested (see row 9 in Table 1). Because all of the measurements show robust and consistent behavior, camera gain was not considered an influential factor in data analysis.

Since the camera sensor recorded 20x640 $=12800$ values of the light intensities across its sensor area, we used pixel by pixel data and show the mean values and the spread (see the rows 1 through 6 in Table 1). Figure 6 presents a representative measurement of the mean beam intensities across the sensor area and instantaneous values of one pixel collected during a twominute run. Figure 7 shows intensity variation across the sensor and over the time. Despite the variations in measured intensities, we were able to predict intensity values for the combined $\mathrm{HV}$ beam fairly successfully to within $2 \%$ (see the rows 7 and 8 in Table 1).

Table 1. Summary of measurements for BGSM beams taken on Dec. 17 2014. The conditions for all of the measurements were approximately the same and the tests were performed consecutively.

\begin{tabular}{|c|c|c|c|c|c|c|}
\hline & $\begin{array}{l}\text { AVERAGE INTENSITY AND STANDARD } \\
\text { DEVIATION OF MEASUREMENTS }\end{array}$ & & & & & \\
\hline 1 & Measured Horizontal Intensity & 131 & 167 & 166 & 200 & 242 \\
\hline 2 & $S T D$ & 22 & 29 & 29 & 34 & 42 \\
\hline 3 & Measured Vertical Intensity & 126 & 162 & 157 & 188 & 236 \\
\hline 4 & $S T D$ & 22 & 29 & 29 & 33 & 41 \\
\hline 5 & Measured Combined HV beam Intensity & 257 & 327 & 319 & 392 & 475 \\
\hline 6 & $S T D$ & 45 & 60 & 56 & 69 & 85 \\
\hline 7 & Predicted Combined HV beam Intensity & 257 & 329 & 324 & 388 & 478 \\
\hline 8 & Fractional Error in prediction & 0.001 & 0.006 & 0.013 & 0.012 & 0.006 \\
\hline \multirow[t]{2}{*}{9} & Camera Gain & 150 & 200 & 200 & 250 & 300 \\
\hline & $\begin{array}{l}\text { AVERAGE SCINTILLATION INDEX AND } \\
\text { STANDARD DEVIATION OF MEASUREMENTS }\end{array}$ & & & & & \\
\hline 10 & Measured Horizontal Scintillation index & 0.909 & 0.936 & 0.913 & 0.912 & 0.913 \\
\hline 11 & $S T D$ & 0.0072 & 0.0069 & 0.0094 & 0.0075 & 0.0094 \\
\hline 12 & Measured Vertical Scintillation index & 0.879 & 0.902 & 0.878 & 0.888 & 0.900 \\
\hline
\end{tabular}




\begin{tabular}{|l|l|l|l|l|l|l|}
\hline 13 & STD & 0.0082 & 0.0096 & 0.0102 & 0.0068 & 0.0065 \\
\hline 14 & Measured Combined HV beam Scintillation index & $\mathbf{0 . 4 4 7}$ & $\mathbf{0 . 4 4 2}$ & $\mathbf{0 . 4 4 8}$ & $\mathbf{0 . 4 4 2}$ & $\mathbf{0 . 4 4 6}$ \\
\hline 15 & $S T D$ & 0.0026 & 0.0025 & 0.0023 & 0.0025 & 0.0026 \\
\hline 16 & Predicted Combined HV beam Scintillation index & 0.448 & 0.460 & 0.448 & 0.451 & 0.453 \\
\hline 17 & Fractional Error in prediction & 0.001 & 0.038 & 0.001 & 0.020 & 0.017 \\
\hline 18 & Combined Scintillation index reduction \% & $\mathbf{5 0}$ & $\mathbf{5 2}$ & $\mathbf{5 0}$ & $\mathbf{5 1}$ & $\mathbf{5 1}$ \\
\hline
\end{tabular}

The second segment of the Table 1 summarizes the scintillation index measurements and calculations. Equation 2.3 was used in rows 10, 12 and 14 and equation 2.5 was used in row 18. Of note is the excellent repeatability of the values in the presence of widely varying intensities observed in the rows 1 through 6.

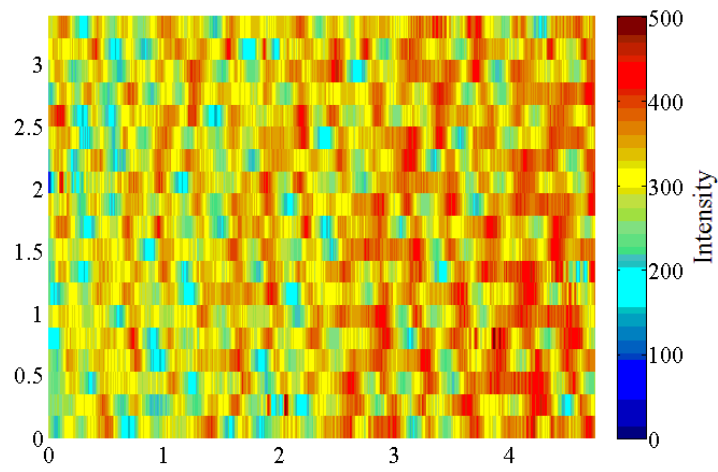

a)

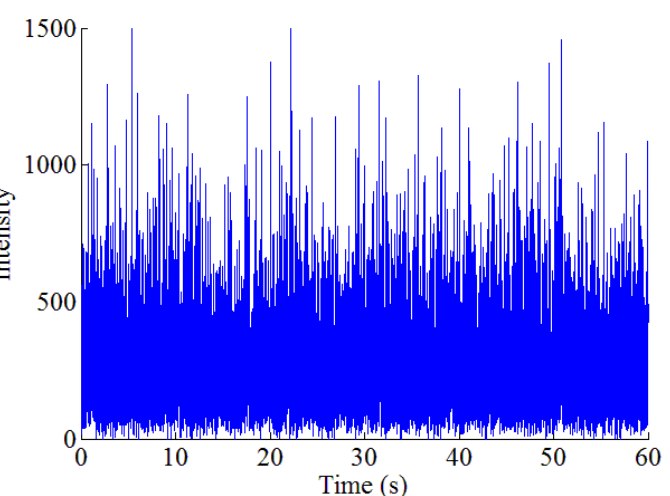

b)

Fig. 6. a) Mean intensity spatial distribution across the sensor area, position in $\mathrm{mm}$. b) Instantaneous beam intensity over the course of the observation time at a representative pixel. Sensor area dimensions are given in $\mathrm{mm}$.

Since the cycling and recording rates were matched, the measured scintillation index for vertically and horizontally polarized beams is at the order of 1 as expected. The measured scintillation index for the electromagnetic (HV) beams and its theoretical prediction match remarkably to better than $4 \%$. We obtained measurements where the scintillation of the vertically polarized beam was dominant and still the predictions matched. The robustness of this result is significant for further applications.

Figure 7 shows the independence of scintillation index on measured light intensity. Standard deviation of the scintillation index across the sensor is remarkably small. Figure 8 shows the spatial distribution of the scintillation index for all runs given in Table 1. Line 15 of Table 1 shows the standard deviation of scintillation across the sensor area. Note the different 
scintillation pattern between the Gaussian beam effectively measured in Fig. 5 and the distribution for the partially coherent beams in Fig. 8.

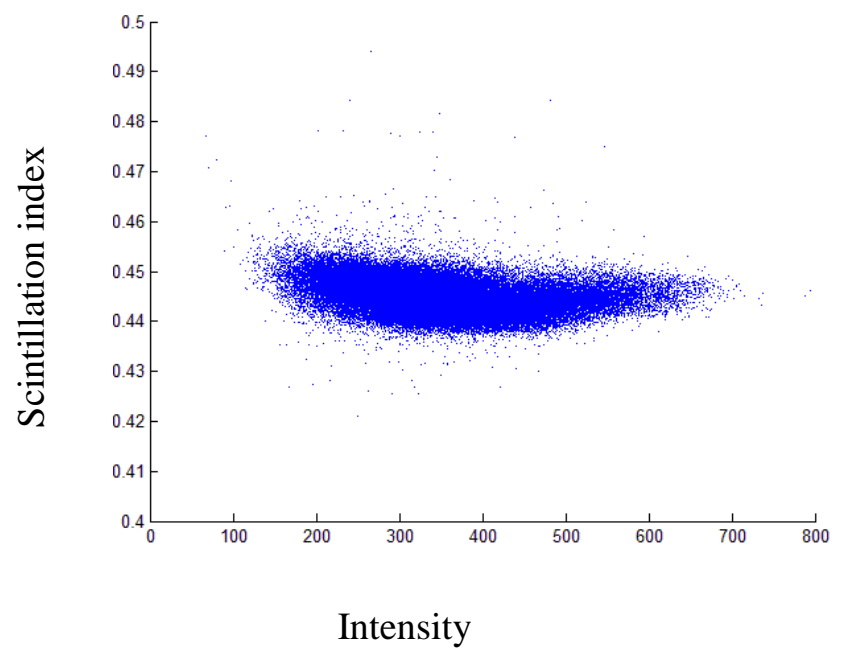

Fig. 7. Correlation between measured scintillation index and measured light intensity for each pixel of BGSM beams as given in the Table 1.
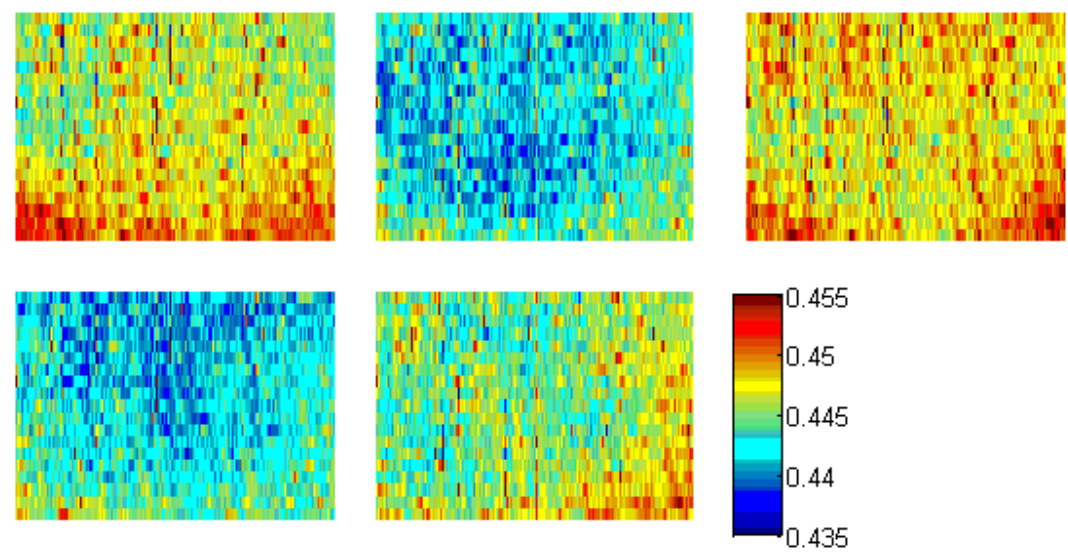

Fig. 8. Measured scintillation index spatial distribution across the sensor area for the beams presented in the same order as given in Table 1. Sensor dimensions are the same as in Fig 6. Vertical and horizontal polarizations have very similar realizations and are not included for brevity.

Most of the results in the paper present spatial distributions across the camera sensor area. The values have been calculated for each pixel. To validate the pixel-by-pixel spatial representation approach, the change of the scintillation index as a function of observation area was explored as shown in Figure 9(a). The scintillation index was calculated for the indicated averaged pixel number for all of the experiments given in Table 1. After an initial slight dip, where up to 10 pixels are averaged, the scintillation index is slowly linearly changing for up to the full frame. The investigation noticeably shows that the scintillation index does not significantly depend on the size of the observation area. The relative change is in the order of $3 \%$ for the aperture averaging with diameter of $9 \mathrm{~mm}$. This effect can be compared relative to time 
binning [see Fig. 19 (b)] where the scintillation index changes several orders of magnitude with longer observation times.

The absolute value of scintillation index depends on the camera capture rate and the SLM screen cycling rate and in particular their ratio. In our experiments, the camera capture rate of $\sim 300 \mathrm{~Hz}$ and phase screen cycling rate on the SLMs was $333 \mathrm{~Hz}$. We simulated different capture rates by temporal binning as given in Sec. 4. Figure 9(b) demonstrates the change of the scintillation as the function of temporal binning. Our simulation validates that independent of the actual capture and cycle rates the relative scintillation reduction is preserved.

If we spatially average the sensor area of $0.02 \mathrm{~mm}$, which corresponds to $\sim 10$ pixels spatial distribution of the intensities is given in Fig.10. It is clearly demonstrated that BGSM beams have sloped intensities.

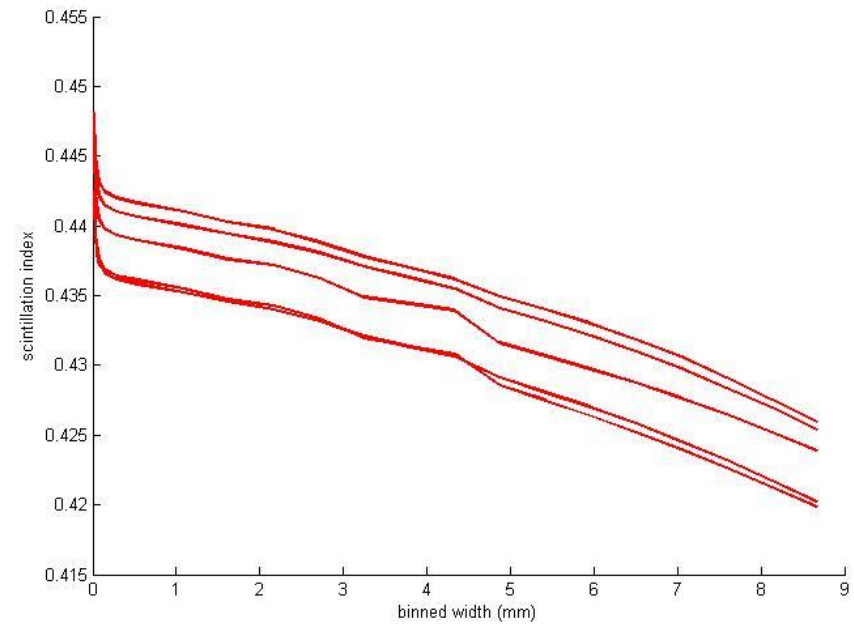

a)

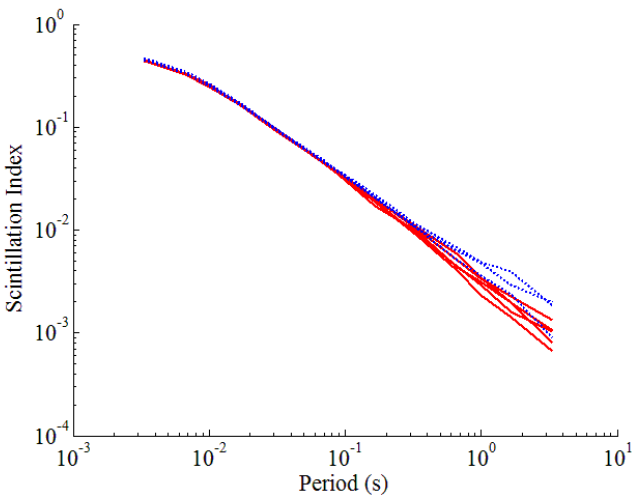

b)

Figure 9. a) Spatial binning and b) Temporal binning for the beams given in Table1. Solid red lines are the ring beams and dotted blue lines are the MGSM beams.
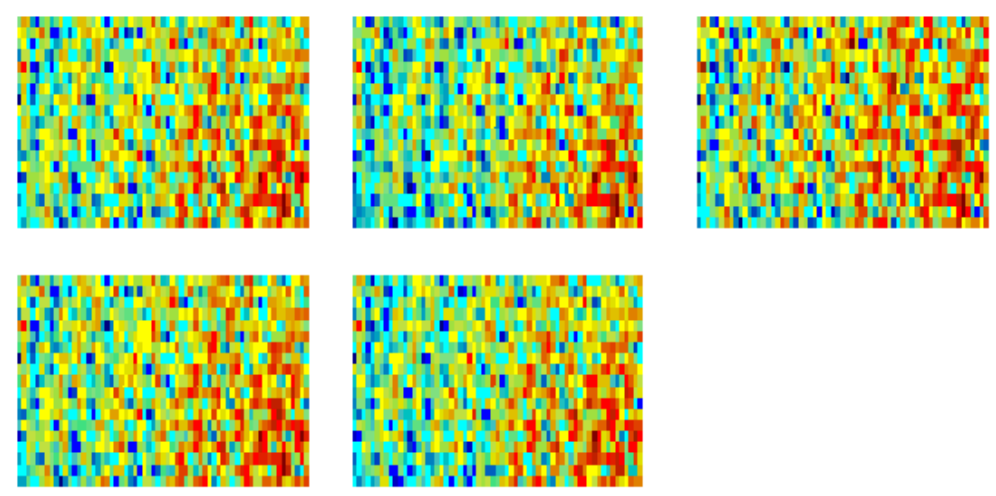
Fig. 10. Spatial distribution of measured intensity across the sensor area for the beams given in the Table 1. Spatial a

veraging of 10 pixels applied. Note that the intensities are normalized in order to demonstrate qualitatively the slope of the intensities for the BGSM beams.

\section{Conclusions}

This paper presents a comprehensive experimental analysis of the scintillation index in the case of propagating Bessel-Gaussian Schell Model electromagnetic beams in weak atmospheric turbulence. The in-depth discussion of the measurement procedure and data processing provides reliability of the experiments taken. We experimented with BGSM beams that were vertically and horizontally polarized as well as combined to create electromagnetic beams.

The main contribution of our investigation is the verification of the demonstration of scintillation index reduction by up to $50 \%$ when scalar spatially pseudo partially coherent beams are compared to electromagnetic beams with uncorrelated field components. Moreover, this reduction is not influenced by atmospheric turbulence, at least in its weak fluctuating regime, as was shown by propagation of the beam in actual atmospheric channel, the result being extended from a previous one obtained for hot-air turbulent emulator characterized in [34]. As we have experimentally shown in [34] and in this paper, the fundamental result of 50\% scintillation reduction holds regardless of the source correlation function shape and the turbulent environment.

\section{Acknowledgement}

S. Avramov-Zamurovic and S. Guth are supported by US ONR grant: N0001414-WX-00267. R. Malek-Madani is supported by US ONR grant N0001414WX00197. C. Nelson' is supported by US ONR grant N001614WX30023 . O. Korotkova is supported by theAir Force Office of Scientific Research (AFOSR) grant FA9550-12-1-0449); and the Office of Naval Research (ONR) grant N00014-15-1-2350.

\section{References}

[1] Carl Leader J. "Intensity fluctuations resulting from partially coherent light propagating through atmospheric turbulence”, J. Opt. Soc. Am. 1979;69:73-84.

[2] Banakh VA, Buldakov VM. "Effect of the initial degree of spatial coherence of a light beam on intensity fluctuations in a turbulent atmosphere", Opt. Spectrosc. 1983;55:707-712.

[3] Ricklin JC, Davidson FM. "Atmospheric turbulence effects on a partially coherent Gaussian beam: implications for free-space laser communication”, J. Opt. Soc. Am. A. 2002;19:1794-1802. 
[4] Ricklin JC, Davidson FM. "Atmospheric optical communication with a Gaussian Schell beam”, J. Opt. Soc. Am. A. 2003;20:856-866.

[5] Korotkova O, Andrews LC, Phillips RL. "Model for a partially coherent Gaussian beam in atmospheric turbulence with application in lasercom”, Opt. Eng. 2004;43:330-341.

[6] Churnside JH. "Aperture averaging of optical scintillations in the turbulent atmosphere", Appl.Opt. 1991;30:1982-1994.

[7] Rosenberg S, Teich MC. "Photocounting array receivers for optical communication through the lognormal atmospheric channel 2: optimum and suboptimum receiver performance for binary signaling", Appl. Opt. 1973;12:2625-2634.

[8] Lee EJ, Chan VWS. "Part 1: optical communication over the clear turbulent atmospheric channel using diversity”, IEEE J. Select. Areas Commun. 2004;22:1896-1906.

[9] Berman GP, Bishop AR, Chernobrod BM, Nguyen DC, Gorshkov VN. "Suppression of intensity fluctuations in free space high-speed optical communication based on spectral encoding of a partially coherent beam", Opt. Commun. 2007;280:264-270.

[10] Berman GP, Chumak AA. "Influence of phase-diffuser dynamics on scintillations of laser radiation in Earth's atmosphere: long-distance propagation”, Phys. Rev. A. 2009;79:063848063854.

[11] O. Korotkova, "Scintillation index of a stochastic electromagnetic beam propagating in random media" Opt. Comm., 281, 2342-2348 (2008).

[12] Gu Y, Korotkova O, Gbur G. "Scintillation of nonuniformly polarized beams in atmosphericturbulence”, Opt. Lett. 2009;34:2261-2263.

[13] Xiao X, Voelz D. "Wave optics simulation of partially coherent and partially polarized beam propagation in turbulence”, Proc. SPIE 7464, Free-Space Laser Communications IX, 74640T;2009 Aug 21; San Diego, CA.

[14] S. Avramov-Zamurovic, C. Nelson, R Malek-Madani, O. Korotkova, "Polarization-induced reduction in scintillation of optical beams propagating in simulated turbulent atmospheric channels", Waves in Random and Complex Media, Vol. 24, (4), pp 452-462, (2014).

[15] Sahin S, Korotkova O. "Light sources generating far fields with tunable flat profiles", Opt. Lett. 2012;37:2970-2972.

[16] Korotkova O, Sahin S, Shchepakina E. "Multi-Gaussian Schell-model beams", J. Opt. Soc.Am. A. 2012;29:2159-2164.

[17] Mei Z, Korotkova O, Shchepakina E. "Electromagnetic multi-Gaussian Schell-model beams", J. Opt. 2013;15:025705. 
[18] Hyde IV M V, Basu S, Xiao X and Voelz D G, "Producing any desired far-field mean irradiance pattern using a partially-coherent Schell-model source", J. Opt. 17, 055607 (2015).

[19] Wang F, Liang C, Yuan Y, and Cai Y, "Generalized multi-Gaussian correlated Schellmodel beam: from theory to experiment", Opt. Express 23456-23464 (2014)

[20] Z. Mei, O. Korotkova, "Random sources generating ring-shaped beams", Optics Letters, Vol. 38, (2), pp. 91-93 (2013)

[21] Cang J., Xiu P., and Liu X. "Propagation of Laguerre-Gaussian and Bessel-Gaussian Schell-model beams through paraxial optical systems in turbulent atmosphere", Optics \& Laser Technology 54, 35-41(2013).

[22] Reddy SG, Kumar A, Prabhakar S, and Singh RP, "Experimental generation of ring-shaped beams with random sources", Opt. Lett. 38, 4441-4444 (2013).

[23] Davis J. A., Cottrell D. M., Campos J., Yzuel M. J., and Moreno I. "Encoding amplitude information onto phase-only filters", Applied Optics Vol. 38, Issue 23, pp. 5004-5013 (1999)

[24] Gori F. "Matrix treatment for partially polarized, partially coherent beams", Opt. Lett.1998;23:241-243.

[25] Wolf E. "Unified theory of coherence and polarization of random electromagnetic beams", Phys. Lett. A. 2003;312:263-267

[26] Piquero G, Gori F, Romanini P, Santarsiero M, Borghi R, Mondello A. "Synthesis of partially polarized Gaussian Schell-model sources”, Opt. Commun. 2002;208:9-16.

[27] Shirai T, Korotkova O, Wolf E. "A method of generating electromagnetic Gaussian Schellmodel beams”, J. Opt. A: Pure Appl. Opt. 2005;7:232-237.

[28] A. S. Ostrovsky, G. Rodríguez-Zurita, C. Meneses-Fabián, M. Á. Olvera-Santamaría, C. Rickenstorff-Parrao, "Experimental generating the partially coherent and partially polarized electromagnetic source", Opt. Express, Vol. 18, pp 12864-12871 (2010).

[29] Chen Y, Wang F, Liu L, Zhao C, Cai Y, Korotkova O. "Generation and propagation of a partially coherent vector beam with special correlation functions", Phys. Rev. A. 2014;89: 013801.

[30] M. Newberry, Signal to noise considerations for sky subtracted CCD data, Publication of the Astronomical Society of the Pacific, 103, 122:30, Jan. 1991.

[31] G. C. Holst, CCD arrays, cameras, and displays, SPIE optical engineering press, 2nd ed. 1998. 
[32] B. Akselli, A.Kholmatov, H. Nasibov, The use of CCD pixel binning in PIV measurements, Conference: Optomechatronic Technologies, 2009

[33] L. C. Andrews and R. L. Phillips, Laser Beam Propagation through Random Media, 2nd edition, SPIE Press, Bellingham, WA, (2005)

[34] Nelson C, Avramov-Zamurovic S, Malek-Madani R, Korotkova O, Sova R, Davidson F. "Measurements of partially spatially coherent laser beam intensity fluctuations propagating through a hot-air turbulence emulator and comparison with both terrestrial and maritime environments", In: Hemmati H, Boroson DM, editors. Proceedings of SPIE 8610, Free-Space Laser Communication and Atmospheric Propagation XXV, 86100T. San Francisco (CA): SPIE; 2013. 